\title{
Comparison of Salivary Calcium Level in Dentulous and Edentulous Patients
}

\author{
${ }^{1}$ Department of Oral and Maxillofacial Medicine, Faculty of \\ Dentistry, Guilan University of Medical Sciences, Rasht, Iran \\ ${ }^{2}$ Department of Prostodontics, Faculty of Dentistry, Guilan \\ University of Medical Sciences, Rasht, Iran
}

Maryam Rabiei ${ }^{1}$ Hamid Neshandar Asli ${ }^{2}$ Mohadeseh Haji Mohamadi²

\begin{abstract}
Address for correspondence Maryam Rabiei, Department of Oral and Maxillofacial Medicine, Faculty of Dentistry, Guilan University of Medical Sciences, Rasht, Iran (e-mail: rabiei@gums.ac.ir).
\end{abstract}

Eur J Dent 2019;13:36-41

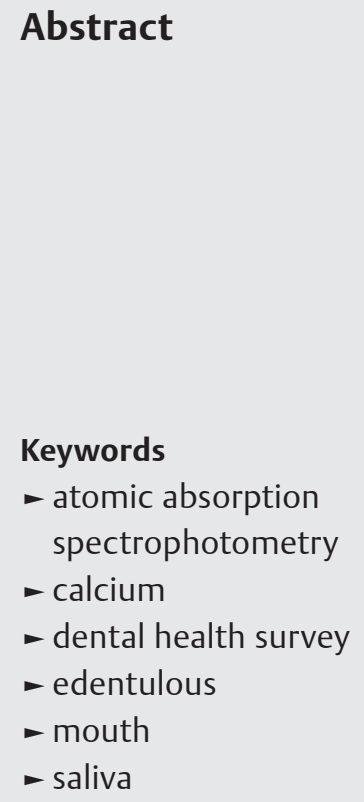

Objectives Calcium is an inorganic component of the saliva, which is especially important in oral and dental health. This study sought to compare unstimulated salivary calcium level of dentulous and edentulous patients.

Materials and Methods This case-control study was conducted on 72 participants, including 36 dentulous and 36 edentulous patients. The unstimulated salivary flow rate of patients and the mean salivary calcium concentration were measured and compared. The data were analyzed using the nonparametric Mann-Whitney U-test.

Results The mean age of participants was 56.3 years. The mean number of teeth was 18.9 in the dentulous group. No significant difference was noted in salivary calcium level of dentulous and edentulous patients. The mean salivary calcium concentration was $0.61 \pm 0.3875 \mathrm{mmol} / \mathrm{L}$ in dentulous and $0.8025 \pm 0.5975 \mathrm{mmol} / \mathrm{L}$ in edentulous patients. Salivary calcium level had a significant inverse correlation with salivary flow rate $(r=-0.370, p=0.027$ ), and by every 1 -unit increase in salivary flow rate, salivary level of calcium significantly decreased by 3.85 units.

Conclusion Edentate had averagely higher salivary calcium level compared to dentulous. The salivary concentration of calcium ion is not correlated to the presence or absence of teeth. Salivary flow rate reduces the calcium concentration.

\section{Introduction}

Saliva and its components play a significant role in oral defense and ecological balance, and in maintaining oral and dental health. ${ }^{1-4}$ Saliva has a complex and unique composition of water, proteins, glycoproteins, and ions responsible for its various properties. ${ }^{1,2}$ Recently, saliva has become an efficient diagnostic tool for scientists in oral and systemic health and diseases. ${ }^{2,3,5}$ The idea of using saliva for diagnostic purposes was first suggested in a late twentieth century. ${ }^{2,6}$ This method is noninvasive, simple, safer, and painless, and does not require a skilled workforce. ${ }^{2,3,7}$ The main electrolytes in the whole saliva include sodium, potassium, calcium, magnesium, chloride, bicarbonate, phosphate, thiocyanate, and fluoride. Of salivary electrolytes, calcium, phosphate, bicarbonate, and fluoride are highly crucial for oral and dental health. ${ }^{8}$ Saliva serves as an irrigating solution for teeth and oral mucosa. It also has the buffering capacity and serves as a reservoir of calcium and phosphate ions, which are required for remineralization of initial enamel lesions. ${ }^{9}$

Calcium is an inorganic component of the saliva, and quantitatively, it is considered as the main mineral component of the human skeletal system. ${ }^{6}$ Calcium plays a significant role in the physiological processes of the human body. Calcium is present in different forms in oral fluid. A large portion of salivary calcium ions is bonded to inorganic components such as orthophosphate or carbonate or macromolecules such as proteins. Almost half the calcium present in the saliva is in ionized form. Most studies have shown that the concentration of calcium present in dental plaque is significantly higher than that in the saliva. ${ }^{10}$ The concentration of calcium ion in the saliva is much lower than its plasma concentration., ${ }^{2,37}$

Hormonal factors can affect saliva composition in the long term. For instance, the salivary concentration of calcium 
ions is higher in the mid-menstrual period compared to premenstrual and postmenstrual phases. ${ }^{11}$ Estrogen plays an important role in calcium metabolism in menopausal women by inhibiting bone decalcification. ${ }^{5}$ Saliva also protects the tooth structure by providing calcium and phosphate ions. According to the chemical law of mass action, these ions affect driving forces responsible for deposition or dissolution of calcium hydroxyapatite, which is the primary mineral component of dental hard tissue. ${ }^{12,13}$

Change in the level of calcium present in the fluid surrounding enamel crystals, which are mainly composed of hydroxyapatite, affects the dissolution of minerals by changing the degree of saturation of hydroxyapatite. It appears that the salivary calcium level affects the calcium concentration of dental plaque by the diffusion phenomenon. These changes can consequently impact on the activity of calcium in demineralization and remineralization processes that occur in tooth structure. ${ }^{10}$

In general, low concentration of calcium translates to low thermodynamic driving force for deposition of hydroxyapatite at normal oral $\mathrm{pH}$. Driving force for dissolution of hydroxyapatite is higher at $\mathrm{pH}$ values lower than the critical $\mathrm{pH}^{12}$ According to Dawes, calcium is the only salivary electrolyte at a constant level. In contrast to other salivary electrolytes, the concentration of calcium is not affected by changes in saliva stimulation. ${ }^{14}$ Dawes presumed that salivary calcium is remaining stable through interaction by teeth surface which undergoes dissolution and deposition to reach to an equilibrium. ${ }^{10}$

This study aimed to compare unstimulated salivary calcium level of dentulous and edentulous patients to find out whether the salivary calcium level remains constant in edentulous patients or not.

\section{Materials and Methods}

The protocol of this case-control study was approved in the Ethics Committee of Guilan University, School of Dentistry (IR.GUMS.REC.1394.463).

Sample size was calculated to be 32 patients in each group for the comparison of salivary calcium level considering $95 \%$ confidence interval $(\mathrm{CI})$ and $80 \%$ study power with expected clinical difference equally for 4 teeth in dichotomous variables (high and low calcium level)..1 Thirty-two dentulous and 32 edentulous patients, matched in terms of age and sex, were selected using convenience sampling and included in the study. Patients were selected among those presenting to the Dental Clinic of Guilan University, School of Dentistry. Patients with osteoporosis, hyperparathyroidism, Paget's disease, renal disorders, and systemic conditions affecting calcium level were not included in the study. Participants were divided into two groups of dentulous and edentulous, and their demographic information and salivary calcium level were assessed. Informed consent was obtained from all individual participants included in the study.

Patients were briefed about the study and written informed consent was obtained from them. Furthermore, participants were provided with a brochure containing information about the study.

\section{Saliva Collection}

Saliva was collected in the morning between 8:30 a.m. and 12:30 p.m. Patients were requested to have breakfast and not to eat or drink anything after that until saliva collection. Before saliva collection, patients were asked to rinse their mouth with deionized water and remove their removable partial or complete denture (if any). Saliva collection tubes and funnels were acid-washed to increase accuracy. Unstimulated saliva was collected in tubes using the spitting method. Salivary flow rate was also measured in gram per minute. The saliva samples were transferred to a laboratory in a cold box. ${ }^{7}$

\section{Measurement of Salivary Calcium Level}

After transfer to a laboratory, the samples were frozen at $-20^{\circ} \mathrm{C}$. Before experiment, the samples were removed from the freezer and thawed to $25^{\circ} \mathrm{C}$. They were then centrifuged at 2,000 $\mathrm{g}$ for $10 \mathrm{~min}$. The samples were transferred to BT3500 autoanalyzer (Biotecnica Instruments, Rome, Italy) for measurement of salivary calcium level, which was determined by colorimetry (atomic absorption spectrometry). In this method, salivary calcium causes a color change when exposed to methylene blue. The degree of color change is proportionate to the concentration of calcium ions. To prevent interference by magnesium ions, 8-hydroxyquinoline is added.

\section{Statistical Analysis}

The data were analyzed using SPSS version 21 (Chicago, Illinois). The Shapiro-Wilk test was used to assess the distribution of unstimulated salivary calcium level in terms of normality in the two groups, which showed that it did not have a normal distribution $(p<0.0001)$. Nonparametric Mann-Whitney U-test was applied to compare the salivary level of calcium between the two groups of dentulous and edentulous patients. Spearman's rho was used to measure the strength of association between salivary calcium and flow rate. Multivariate analysis was exerted for the effect of study group (dentulous/edentulous) on unstimulated salivary calcium.

\section{Results}

This study was conducted on 72 patients in two groups of 36. - Table 1 shows the comparison of unstimulated salivary calcium level, salivary flow rate, and number of teeth in the two groups of dentulous and edentulous patients.

Both groups included 31 males and 5 females. The mean age ( \pm standard deviation) was $56.36 \pm 10.58$ years in dentulous and $56.69 \pm 10.99$ years in edentulous patients.

The results showed that the two groups were not significantly different in terms of unstimulated salivary concentration of calcium $(p>0.05)$. No significant difference was noted in unstimulated salivary calcium level of males $(0.725 \pm 0.525 \mathrm{mmol} / \mathrm{L})$ and females $(0.5625$ $\pm 0.145 \mathrm{mmol} / \mathrm{L})$ either $(p>0.05)$. The mean ( \pm standard deviation) number of teeth in dentulous patients was 18.9 \pm 7.5 teeth (range: $3-30$ ). 
Table 1 Comparison of calcium level, salivary flow rate, and number of teeth in the two groups of dentulous and edentulous patients

\begin{tabular}{|l|l|l|l|}
\hline \multirow{2}{*}{} & \multicolumn{2}{|c|}{ Mean \pm SD } & \multirow{2}{*}{$P$} \\
\cline { 2 - 4 } & Dentulous & Edentulous & \\
\hline Calcium concentration & $0.61 \pm 0.39$ & $0.80 \pm 0.6$ & 0.213 \\
\hline Salivary flow rate & $0.33 \pm 0.20$ & $0.39 \pm 0.25$ & 0.241 \\
\hline Number of teeth & $18.9 \pm 7.5$ & $0 \pm 0$ & 0.000 \\
\hline
\end{tabular}

Abbreviation: SD, standard deviation.

Salivary concentration of calcium was not significantly different in edentulous and dentulous patients $(p>0.05)$. However, in the 50- to 60-year-old group, the mean unstimulated salivary level of calcium was significantly different between edentulous and dentulous patients such that edentulous patients had higher level of unstimulated salivary calcium ( $p=0.04 ;$ - Table 2 ).

According to the Spearman's rho, the results showed that in dentulous patients, unstimulated salivary calcium concentration had an inverse correlation with unstimulated salivary flow rate $(r=-0.370, p=0.027)$ such that by an increase in salivary flow rate, salivary level of calcium decreased. As seen in - Fig. 1, by 1-unit increase in salivary flow rate of dentulous patients, their salivary level of calcium decreased by 3.85 units. The regression formula for this inverse correlation was as follows:

Salivary calcium level $=$ Salivary flow rate $\times(-3.85) 3.85$ $+3.73$

Furthermore, the $R^{2}$ coefficient in - Fig. 1 shows that $24.3 \%$ of changes in unstimulated salivary calcium level in dentulous patients depend on their salivary flow rate; however, no such significant correlations were noted in edentulous patients ( - Fig. 2). There was no correlation between salivary calcium and the number of teeth.

Multivariate analysis of the effect of study group (dentulous/edentulous) on unstimulated salivary calcium level after controlling for the effects of age, sex, and salivary flow rate by multivariate linear regression model with backward stepwise technique and possibility of inclusion and exclusion of variable from the model yielded 0.05 and 0.1 values, indicating that study group $(p=0.05)$ and salivary flow rate $(p=0.038)$ were among the predictors of unstimulated salivary calcium level, such that edentulous patients had averagely higher unstimulated salivary calcium level compared to dentulous patients $(0.9 \pm 0.47)$. Furthermore, by 1 -unit increase in salivary flow rate, after controlling for the effects of age, gender, and study group, unstimulated salivary calcium level decreased by 2.2 units.

The final regression formula for the correlation of unstimulated salivary calcium level and study variables was found to be as follows:

$$
\begin{aligned}
\text { Salivary calcium } & =2.28+0.9 \times(\text { Group } 0=\text { dentulous } / 1 \\
& =\text { edentulous })-2.2 \text { saliva }
\end{aligned}
$$

Multivariate logistic regressions verify the findings. The odds of salivary calcium level up to mean in edentate rising (odds ratio [OR]: 2.6, 95\% CI: 8.16-8.4) versus dentate patients $(p=0.1)$. Salivary flow rate had inverse correlation with salivary calcium level (OR: $0.048,95 \% \mathrm{CI}: 0.002-1.28$ ) $(p=0.07 ;$ - Table 3$)$.

\section{Discussion}

This study aimed to compare unstimulated salivary calcium level in dentulous and edentulous patients. The main finding of this study was the absence of a significant difference in this respect between the two groups.

A previous study showed no significant association between the salivary calcium level and salivary flow rate. ${ }^{14}$ However, calcium ions are in constant reaction with the tooth structure. Thus, in lower salivary flow rate and lower than critical pH, calcium concentration may be different based on the ionization coefficient since salivary calcium constantly reacts with hydroxyapatite in tooth structure. ${ }^{13}$ Another finding of the current study was that the salivary level of this ion was almost similar in dentulous and edentulous patients. It should be noted that due to the absence of teeth in edentulous patients, enamel pellicle and dental plaque do not exist. ${ }^{15} \mathrm{~A}$ reduction in salivary calcium level was noted in

Table 2 Comparison of unstimulated salivary calcium level $(\mathrm{mmol} / \mathrm{L})$ in dentulous and edentulous patients based on the age groups

\begin{tabular}{|l|l|l|l|l|l|}
\hline \multirow{2}{*}{ Age group (years) } & \multicolumn{2}{|c|}{ Mean \pm SD } & Total & $p^{*}$ & \multirow{2}{*}{$p^{* *}$} \\
\cline { 2 - 5 } & Dentulous & Edentulous & & 0.999 & 0.301 \\
\hline$<50$ & $0.68 \pm 0.34$ & $0.68 \pm 0.25$ & $0.68 \pm 0.29$ & 0.04 \\
\hline$>60$ & $0.63 \pm 0.46$ & $1.01 \pm 0.745$ & $0.82 \pm 0.6$ & 0.836 \\
\hline
\end{tabular}

Abbreviation: SD, standard deviation.

*Mann-Whitney.

${ }^{*}$ Kruskal-Wallis. 


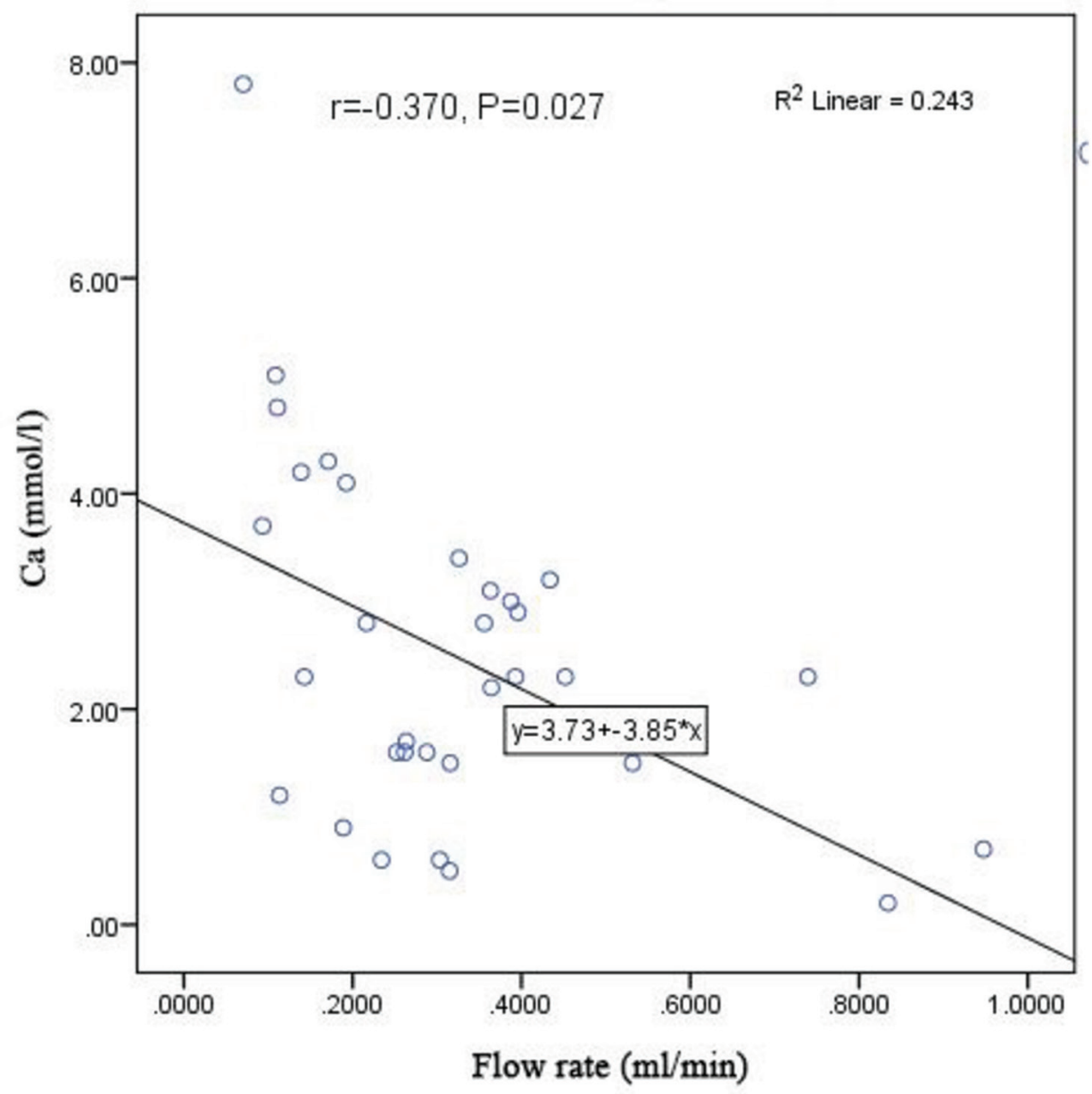

Fig. 1 The scatter plot of the correlation of salivary flow rate with unstimulated salivary calcium level in dentulous patients.

dentulous patients with higher salivary flow rate, which also suggests that the level of calcium ion remains constant. In other words, in higher salivary flow rates, the concentration of calcium decreases in order to maintain a constant level in the saliva. ${ }^{13}$ It should be noted that the increase we are talking about in this study is an increase in unstimulated salivary flow rate. Stimulated saliva is more serous and contains higher levels of proline-rich proteins, statherin, and some other compounds that prevent deposition of calcium on teeth. Different results may be obtained if stimulated saliva is evaluated, which needs further assessment in future studies. In the other hand, point-of-care technology is a potential for biomarker identification through salivary diagnostic toolboxes. ${ }^{16-18}$ This method brings accuracy, repeatability, and reproducibility. This method will be suggested to detecting biomarkers as salivary calcium in further studies. ${ }^{18}$

The absence of any difference in unstimulated salivary calcium concentration of dentulous and edentulous patients suggests many other theories raising the possibility of involvement of many other factors, which may play a role in maintaining this balance. For instance, bicarbonate, phosphate, and urea may have a more significant role in this respect. Enamel pellicle inhibits the uptake of hydrogen ions and prevents the release of calcium and phosphate ions. Saliva is supersaturated with calcium ions compared to hydroxyapatite. In the absence of pellicle, hydroxyapatite crystals tend to expand over the surface (calculus formation). ${ }^{19,20}$ However, it should be noted that some studies have shown a correlation between low concentrations of calcium and phosphate and occurrence of caries, ${ }^{9,21}$ while some others found no such association. ${ }^{22}$ Some authors believe that the role of calcium and phosphate concentrations in this respect is more important than that of $\mathrm{pH}$. Studies have indicated that patients with low concentrations of calcium and phosphate have a critical pH of 6.5, while patients with higher levels of these minerals have a critical pH of 5.5. ${ }^{12}$ Maier et al evaluated the excretion of ionized calcium in pilocarpine-stimulated saliva. The concentration of ionized calcium significantly increased in higher salivary flow rates, but the total calcium concentration had no association with salivary flow rate. ${ }^{19}$ Salivary calcium level of menopausal women with xerostomia can be higher than that of women without xerostomia. $5,6,10,20,23$

Lack of a significant difference in unstimulated salivary calcium level of edentulous and dentulous patients and no association between salivary calcium level and number of teeth in our study question the common belief and the existing literature, stating that individuals with higher salivary calcium level have higher number of sound teeth than those with lower salivary calcium level. ${ }^{17}$ Moreover, the current 


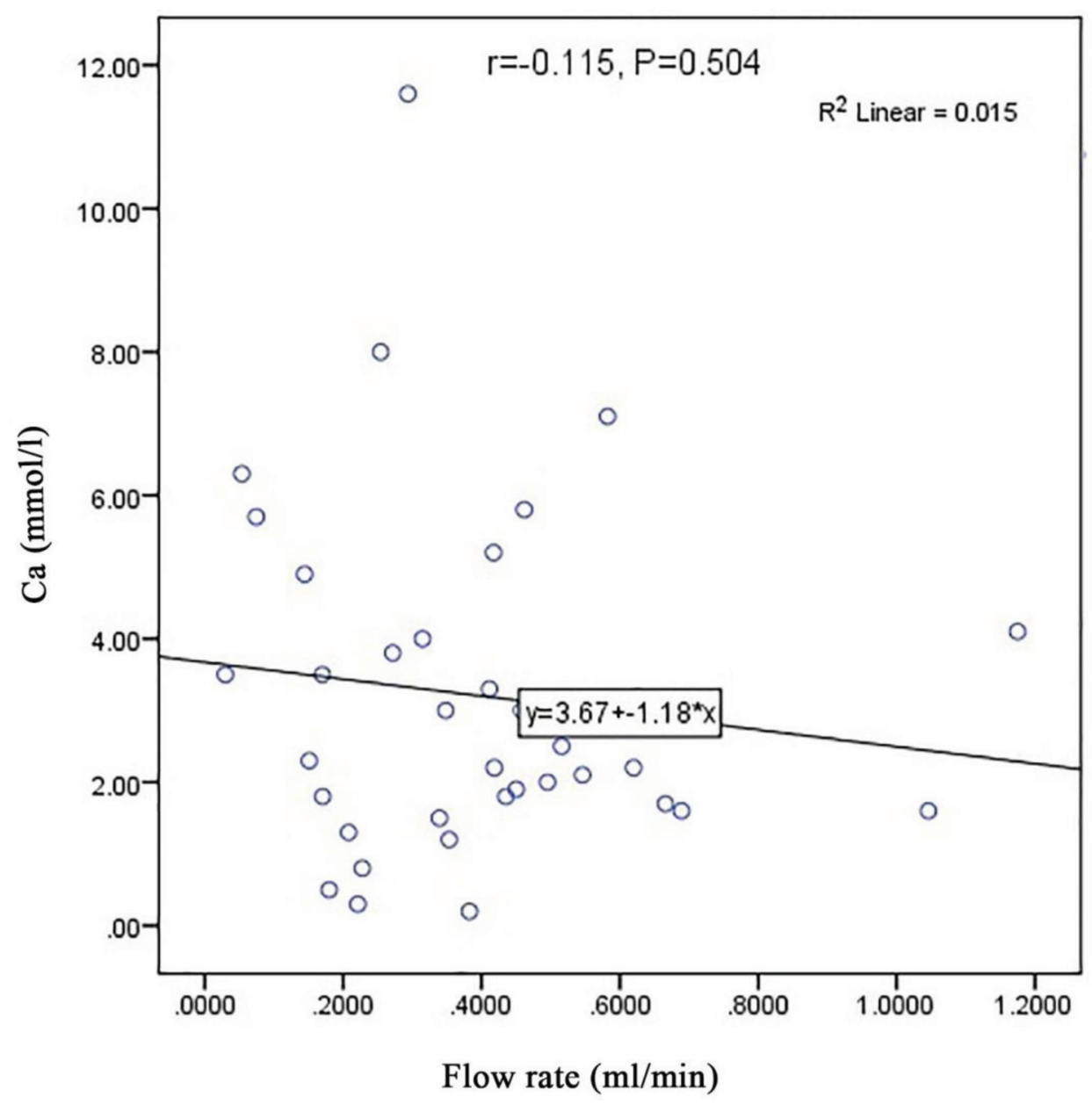

Fig. 2 The scatter plot of the correlation of salivary flow rate with unstimulated salivary calcium level in edentulous patients.

Table 3 Regression coefficients of the predictors of unstimulated salivary calcium level according to the multiple linear regression model with backward stepwise method

\begin{tabular}{|l|l|l|l|l|l|}
\hline \multirow{2}{*}{ Model } & \multicolumn{2}{|l|}{ Unstandardized coefficients } & \multirow{2}{*}{ Significant } & \multicolumn{2}{l|}{$95 \% \mathrm{Cl}$ for $B$} \\
\cline { 2 - 3 } & $B$ & SE & & Lower bound & Upper bound \\
\hline Final model & & & & & \\
\hline Constant & 2.282 & 0.787 & 0.005 & 0.712 & 3.851 \\
\hline Edentulous versus dentulous & 0.900 & 0.468 & 0.058 & -0.033 & 1.833 \\
\hline Salivary flow rate (g/min) & -2.215 & 1.045 & 0.038 & -4.300 & -0.131 \\
\hline
\end{tabular}

Dependent variables are salivary calcium level.

Abbreviations: $\mathrm{Cl}$ : confidence interval; SE: standard error.

study found no significant difference in salivary calcium concentration of males and females or different age groups, which was in contrast to the findings of previous studies. ${ }^{9,11,24,25}$ Rabiei et al suggested the use of increase in salivary calcium concentration higher than the cutoff point of $6.1 \mathrm{mg} /$ $\mathrm{dL}$ for efficient osteoporosis screening of menopausal women. They showed that each 1-unit increase in salivary calcium concentration increased the risk of osteoporosis by 1.6 folds.?

With regard to the critical $\mathrm{pH}$ for enamel dissolution in the oral environment, the reaction between salivary calcium and calcium in tooth structure is a balanced forwardbackward reaction, which constantly changes. When saliva is supersaturated with calcium, its deposition rate exceeds its dissolution rate. When saliva is not saturated with calcium, calcium tends to release from the tooth structure into the saliva and dissolution of minerals occurs at a faster rate. These reactions continue until the saliva is saturated with calcium. At this point, the forward reaction equals the backward reaction. However, on the other hand, the salivary calcium concentration remains constant. 


\section{Conclusion}

The results of this study showed that irrespective of the presence/absence of teeth, salivary calcium level remains constant. It appears that only salivary flow rate can change the salivary concentration of calcium and presence/absence of teeth plays no role in this respect.

\section{Financial Support and Sponsorship}

None.

\section{Conflicts of Interest}

None.

\section{Acknowledgments}

This study was conducted in the Reference Laboratory of Rasht city. The authors would like to thank the laboratory technicians for their sincere cooperation in the conduction of this study.

\section{References}

1 Aliko A, Wolff A, Dawes C, et al. World Workshop on Oral Medicine VI: clinical implications of medication-induced salivary gland dysfunction. Oral Surg Oral Med Oral Pathol Oral Radiol 2015;120(2):185-206

2 Khurshid Z, Zafar MS, Khan RS, Najeeb S, Slowey PD, Rehman IU. Role of salivary biomarkers in oral cancer detection. Adv Clin Chem 2018;86:23-70

3 Khurshid Z, Zohaib S, Najeeb S, Zafar MS, Slowey PD, Almas K. Human saliva collection devices for proteomics: an update. Int J Mol Sci 2016;17(6):E846

4 Mohamed R, Campbell JL, Cooper-White J, Dimeski G, Punyadeera $C$. The impact of saliva collection and processing methods on CRP, IgE, and myoglobin immunoassays. Clin Transl Med 2012;1(1):19

5 Singh R, Pallagatti S, Sheikh S, Singh B, Arora G, Aggarwal A. Correlation of serum oestrogen with salivary calcium in post-menopausal women with and without oral dryness feeling. Gerodontology 2012;29(2):125-129

6 Agha-Hosseini F, Mirzaii-Dizgah I, Moosavi MS. Relationship of serum and saliva calcium, phosphorus and alkaline phosphatase with dry mouth feeling in menopause. Gerodontology 2012;29(2):e1092-e1097

7 Liu J, Duan Y. Saliva: a potential media for disease diagnostics and monitoring. Oral Oncol 2012;48(7):569-577

8 Ahmadi-Motamayel F, Goodarzi MT, Hendi SS, Abdolsamadi $\mathrm{H}$, Rafieian N. Evaluation of salivary flow rate, $\mathrm{pH}$, buffering capacity, calcium and total protein levels in caries free and caries active adolescence. J Dent Oral Hyg 2013;5(4):35-39
9 Matsuo S, Lagerlöf F. Relationship between total and ionized calcium concentrations in human whole saliva and dental plaque fluid. Arch Oral Biol 1991;36(7):525-527

10 Kavanagh DA, Svehla G. Variation of salivary calcium, phosphate and buffering capacity in adolescents. Arch Oral Biol 1998;43(12):1023-1027

11 Anderson P, Hector MP, Rampersad MA. Critical pH in resting and stimulated whole saliva in groups of children and adults. Int J Paediatr Dent 2001;11(4):266-273

12 Dawes $\mathrm{C}$. What is the critical $\mathrm{pH}$ and why does a tooth dissolve in acid? J Can Dent Assoc 2003;69(11):722-724

13 Rabiei M, Masooleh IS, Leyli EK, Nikoukar LR. Salivary calcium concentration as a screening tool for postmenopausal osteoporosis. Int J Rheum Dis 2013;16(2):198-202

14 Dawes C. Salivary flow patterns and the health of hard and soft oral tissues. J Am Dent Assoc 2008;139(Suppl):18S-24S

15 Sewón LA, Karjalainen SM, Söderling E, Lapinleimu H, Simell O. Associations between salivary calcium and oral health. J Clin Periodontol 1998;25(11 Pt 1):915-919

16 Khan RS, Khurshid Z, Yahya Ibrahim Asiri F. Advancing point-of-care (PoC) testing using human saliva as liquid biopsy. Diagnostics (Basel) 2017;7(3):E39

17 Aro K, Wei F, Wong DT, Tu M. Saliva liquid biopsy for point-ofcare applications. Front Public Health 2017;5:77

18 Khurshid Z. Salivary point-of-care technology. Eur J Dent 2018;12(1):1-2

19 Maier H, Triebel C, Heidland A. The flow-rate-dependent excretion of ionized calcium in pilocarpine-stimulated human submandibular saliva. Arch Oral Biol 1983;28(10):907-909

20 Singh B, Sheikh S, Pallagatti S, Kaur K, Sohi R. Evaluation of salivary calcium and salivary parathyroid levels in postmenopausal women with and without oral dryness. Contemp Clin Dent 2013;4(4):488-492

21 Agha-Hosseini F, Mirzaii-Dizgah I, Moghaddam PP, Akrad ZT. Stimulated whole salivary flow rate and composition in menopausal women with oral dryness feeling. Oral Dis 2007;13(3):320-323

22 Sewón L, Laine M, Karjalainen S, Leimola-Virtanen R, Hiidenkari T, Helenius H. The effect of hormone replacement therapy on salivary calcium concentrations in menopausal women. Arch Oral Biol 2000;45(3):201-206

23 Sewón L, Laine M, Karjalainen S, Doroguinskaia A, Lehtonen-Veromaa M. Salivary calcium reflects skeletal bone density of heavy smokers. Arch Oral Biol 2004;49(5):355-358

24 Sewón L, Mäkelä M. A study of the possible correlation of high salivary calcium levels with periodontal and dental conditions in young adults. Arch Oral Biol 1990;35(Suppl):211S-212S

25 Larsen MJ, Jensen AF, Madsen DM, Pearce EI. Individual variations of $\mathrm{pH}$, buffer capacity, and concentrations of calcium and phosphate in unstimulated whole saliva. Arch Oral Biol 1999;44(2):111-117 